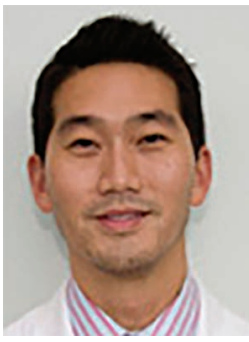

Roderick Tung, M.D.

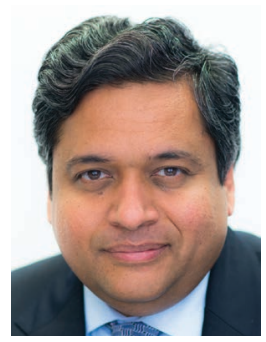

Kalyanam Shivkumar M.D., Ph.D.

\section{EPICARDIAL ABLATION OF VENTRICULAR TACHYCARDIA}

\author{
Roderick Tung, M.D.; Kalyanam Shivkumar, M.D., Ph.D. \\ UCLA Health System, David Geffen School of Medicine at UCLA, Los Angeles, California
}

\title{
Abstract
}

Epicardial mapping and ablation via a percutaneous subxiphoid technique has been instrumental in improving the working understanding of complex myocardial scars in various arrhythmogenic substrates. Endocardial ablation alone may not be sufficient in patients with ischemic cardiomyopathy, dilated cardiomyopathy, arrhythmogenic right ventricular cardiomyopathy, hypertrophic cardiomyopathy, and Chagas disease to prevent recurrent ventricular tachycardia. Multiple observational studies have demonstrated greater freedom from recurrence with adjunctive epicardial ablation compared with endocardial ablation alone. While epicardial ablation is performed predominantly at tertiary referral centers, knowledge of the technical approach, clinical indications, and potential complications is imperative to maximizing clinical success and patient safety.

In 1996, Sosa and colleagues modified the pericardiocentesis technique to enable percutaneous access to the pericardial space for mapping and catheter ablation of ventricular tachycardia. ${ }^{1}$ Originally developed for patients with epicardial scarring due to chagasic cardiomyopathy and patients with ischemic cardiomyopathy refractory to endocardial ablationm, ${ }^{2,3}$ this approach has since become an essential part of the armamentarium for the treatment of ventricular tachycardia. Myocardial scars are three-dimensionally complex with varying degrees of transmurality, and the ability to map and ablate the epicardial surface has contributed to a greater understanding of scar-related VT in postinfarction cardiomyopathy and nonischemic substrates including idiopathic dilated cardiomyopathy, arrhythmogenic right ventricular cardiomyopathy, hypertrophic cardiomyopathy, and chagasic cardiomyopathy. In this review, we highlight the percutaneous approach and discuss clinical indications and potential complications.

\section{Indications for Epicardial Mapping and Ablation}

Epicardial ablation is most commonly employed after failed endocardial ablation. Additionally, it may be considered based on a high pretest probability of midmyocardial or subepicardial scar, which is commonly seen in patients with arrhythmogenic right ventricular cardiomyopathy (ARVC), dilated cardiomyopathy (DCM), and hypertrophic cardiomyopathy (HCM) ${ }^{4,5}$ Magnetic resonance imaging and electrocardiographic criteria may be clinically useful when assessing for the presence of epicardial substrate (Figure 1). As postinfarct scar follows a physiologic pattern of subendocardial necrosis that progresses towards the epicardium, it has been commonly thought that epicardial ablation has a lower yield in patients with ischemic cardiomyopathy. ${ }^{6,7}$

The use of epicardial ablation as a preemptive and adjunctive strategy has been reported in multiple observational studies of DCM, ARVC, and HCM. ${ }^{8-12}$ A combined epicardial-endocardial approach is associated with improved freedom from ventricular tachycardia (VT) recurrence when compared to more limited endocardial strategies. ${ }^{13}$ In our experience, patients with ischemic cardiomyopathy also experience greater freedom from recurrence when epicardial ablation is performed in combination with endocardial ablation (Figure 2). ${ }^{14}$ The yield of epicardial ablation in the postinfarct setting is dependent on prior ablation history, where enriched epicardial substrates may be selected due to referral bias after unsuccessful endocardial ablation. ${ }^{15,16}$

\section{Subxiphoid Percutaneous Technique}

Prior to attempting access, anticoagulants, antithrombotics, and antiplatelet agents should be discontinued to minimize the risk of bleeding, and a typed blood sample should be obtained. Surgical backup is important for centers attempting epicardial ablation in the event of complications. ${ }^{17}$ Epicardial access is obtained prior to systemic heparinization, and we often perform epicardial mapping prior to endocardial mapping to minimize the total duration of systemic anticoagulation during the procedure. It is our opinion that epicardial access performed under general anesthesia optimizes patient safety, comfort, and overall success. However, general anesthesia may impair VT inducibility and prolong procedural duration. If conscious sedation is chosen, the sedation may be deepened for the puncture and lightened after successful access is obtained.

The procedure is started with sterile preparation of the subxiphoid region, which is then anesthetized with topical $1 \%$ lidocaine. Antibiotics are administered to minimize the risk of bacterial pericarditis. At our center, the puncture site is performed one fingerbreadth left of the subxiphoid process, allowing for access through Larrey's space (trigonum sternocostale), which is reported to be avascular. ${ }^{17,18}$ A 17 G epidural needle allows an angled guidewire course away from the myocardium after the parietal pericardium is punctured. Two lengths of Tuohy epidural needles are available at our institution: $17 \mathrm{G}$ x 3.5 in $(90 \mathrm{~mm}, \mathrm{BD}$ 


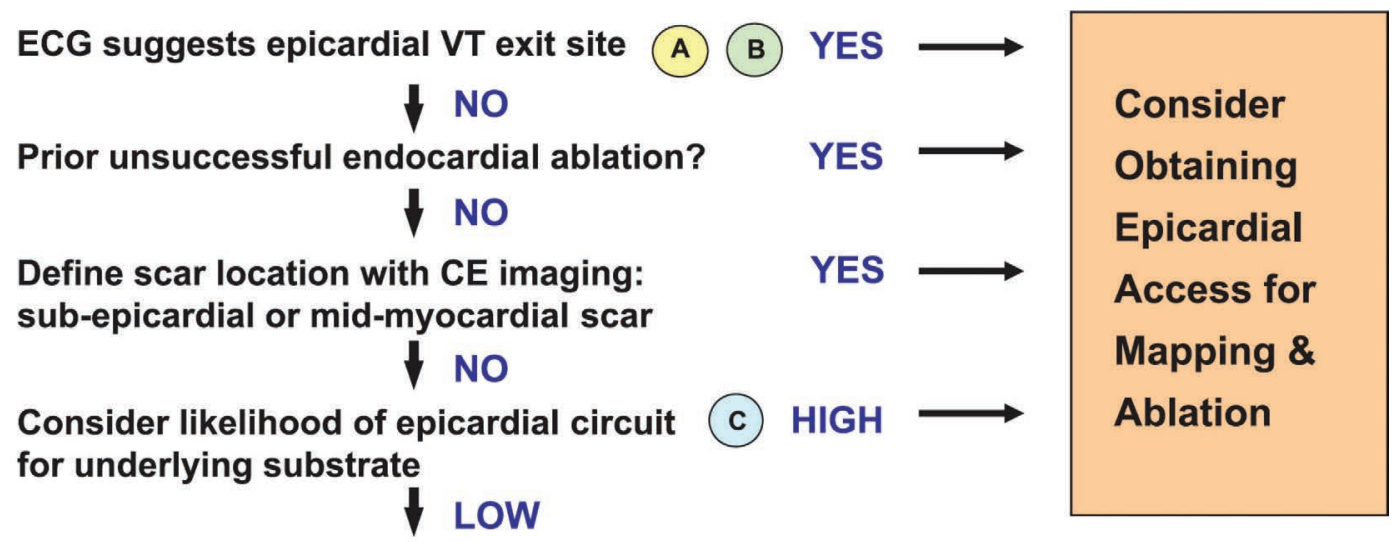

Perform Endocardial Mapping and Ablation
(A)

\begin{tabular}{|l|}
\hline \multicolumn{1}{|c|}{ ECG Criteria } \\
\hline 1) Pseudo-delta $>34 \mathrm{~ms}$ \\
2) IDT (V2) $>85 \mathrm{~ms}$ \\
3) Shortest RS complex $>121 \mathrm{~ms}$ \\
4) ORS duration $>211 \mathrm{~ms}$ \\
\hline
\end{tabular}

(B)

1) Absence of inferior $Q$ wave

2) Pseudodelta $\geq 75 \mathrm{~ms}$

3) $\mathrm{MDI}>0.59$

4) Presence of $Q$ wave in lead I
ECG Criteria for NICM

Medical, Franklin Lakes, NJ) and 17 G x 6 in (152 mm, Hakko Co, Ltd., Tokyo, Japan). A shallow entry initially directed to the left shoulder is preferred to minimize puncture of the liver, with an increase toward $45^{\circ}$ after several centimeters.

Biplane fluoroscopy is useful to assess the approach of the needle for both the anterior and posterior (inferior) approaches. A helpful rule of epicardial access is to puncture at a site remote from the anticipated scar region since acute curves required to map the region around the puncture site are technically challenging. If an anterior puncture is desired, the needle track is continued along a shallow course $\left(<30^{\circ}\right)$, and a steep lateral projection helps assess the needle track just posterior to the sternum as it approaches the anterior right ventricle. A posterior approach requires steepening the needle angle $\left(>45^{\circ}\right)$ to aim for the basal portion of the heart, which is fluoroscopically marked by the coronary sinus catheter. The right anterior oblique projection is useful for determining a basal versus apical approach angle. The left anterior oblique (LAO) projection is helpful to identify the right ventricular free wall that forms the right heart border (Figure 3). We prefer to approach with an angle that is more tangential to the inferior heart border visualized on LAO to minimize right ventricular puncture. However, a puncture directed too septally increases the risk of posterior descending artery or middle cardiac vein injury. The incidence of diaphragmatic and hepatic puncture is higher with a posterior approach, but we have not experienced any adverse clinical events.

Once the needle is advanced to the inferior heart border, cardiac pulsations, a current of injury detected with alligator clips, or ventricular ectopy can all help signify contact with the parietal pericardium. Cardiac pulsations should be assessed during inspiration as the downward displacement of the heart puts it in closer proximity to the needle. The minimum amount of contrast is injected to visualize "tenting" or indentation of the fibrous
(C)

\begin{tabular}{|lr|}
\hline Probability of Epicardial & Focus \\
\hline Normal & $6 \%$ \\
ICM & $16 \%$ \\
NICM & $35 \%$ \\
ARVC & $41 \%$ \\
Other CM & $18 \%$ \\
& \\
\hline
\end{tabular}

pericardium since larger quantities of contrast may obscure the fluoroscopic field of view (Figure 4). Once the pericardium is in contact with the needle, puncture is performed and may be felt as a "pop" or, more commonly, visualized with release of the contrast-stained pericardial outer surface. A slight withdrawal of the needle once puncture is sensed, as the pericardium releases, reduces the risk of cardiac perforation as the advanced needle frequently violates the anatomic plane of the right ventricle. Contrast is quickly injected to confirm layering within the pericardial space, and a guidewire is advanced along the lateral heart border in LAO projection. The guidewire must be confirmed to reach the left heart border in LAO view to exclude puncture of the right ventricle. The optimal guidewire course traverses several chambers and reaches the outermost portion of the fluoroscopic cardiac silhouette. Following this, we use a smaller soft-tip dilator ( $5 \mathrm{Fr}$ ) over the wire for further confirmation. The first aspirate of pericardial fluid is obtained via this small dilator to assess for bleeding, and 10 to $20 \mathrm{cc}$ of contrast is injected.

Contrast pericardiography is helpful to confirm the pericardial puncture and assess for adhesions, which may limit the ability to navigate the catheter and increase the probability of pericardial bleeding. Once this is performed, a long wire is placed across the transverse sinus or anterior to the great vessels, and a long sheath is advanced under LAO projection. We have chosen a long sheath at our center to increase the distance of the operator from the fluoroscopy tube and to minimize the risk of losing access. A steerable sheath can be used if small adhesions are encountered or to assist in mapping regions that are not easily accessible with a fixed sheath. ${ }^{19}$ Additionally, we always employ a "double wire" technique to retain one wire in the case of inadvertent sheath withdrawal during mapping.

In patients who had prior cardiac surgery, dense adhesions decrease the operator's ability to determine if the puncture is 


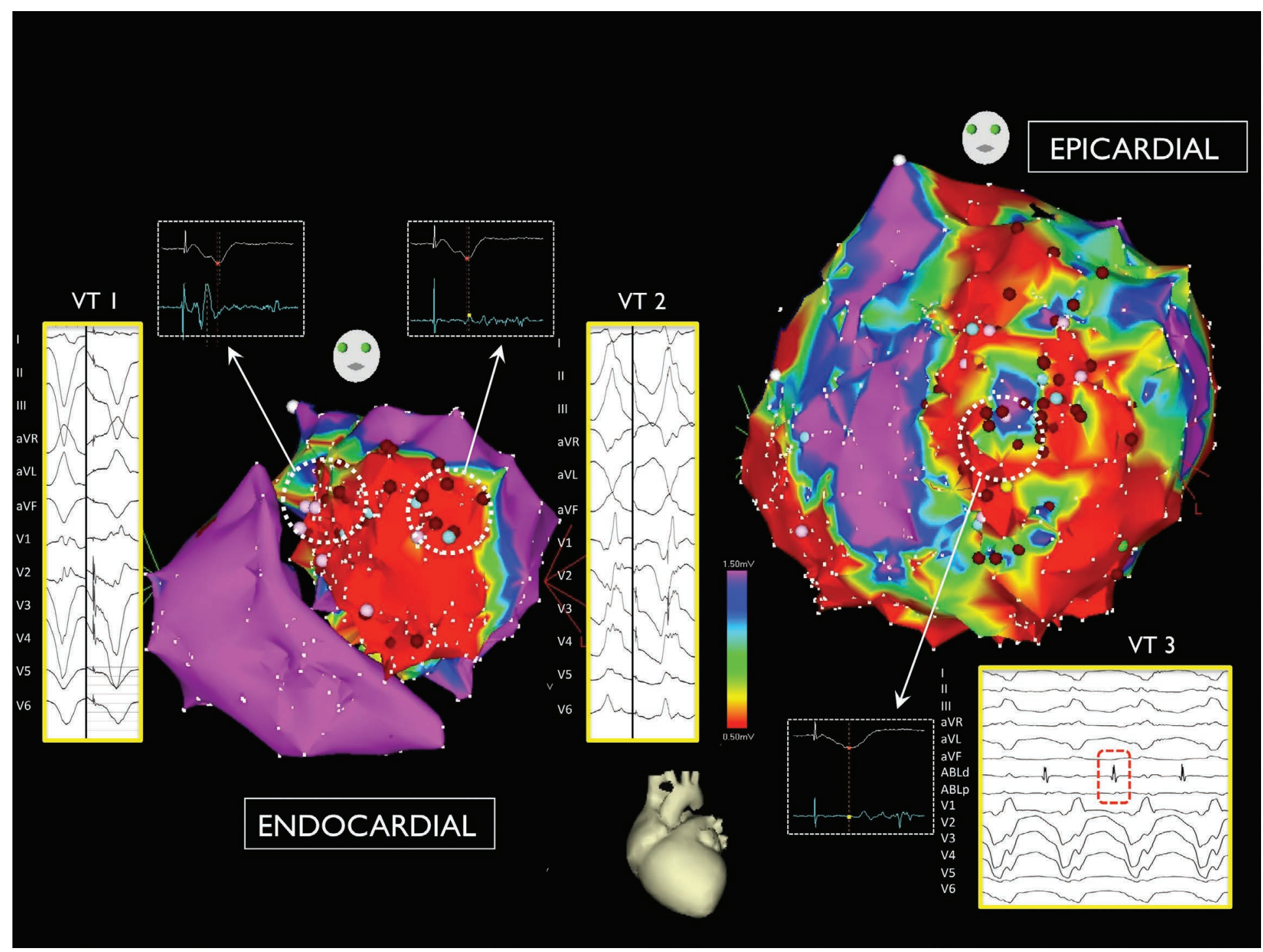

Figure 2. Combined epicardial and endocardial mapping of anterior infarction in a patient with multiple ventricular tachycardias. Termination of a third morphology was achieved with ablation on the epicardium. Modified from Tung et al. Heart Rhythm. 2013;10:490-8.

in the pericardial space, limit the ability to access all epicardial regions, and increase the risk of bleeding as adhesions are bluntly dissected without direct visualization. ${ }^{20}$ Although percutaneous access has been reported in such patients, it is our practice not to attempt percutaneous access due to limited access and an increased risk of complications. Rather, minimally invasive surgical access can be obtained via a subxiphoid window or limited anterior thoracotomy to facilitate catheter access in patients who underwent prior cardiac surgery..$^{21,22}$

\section{Collateral Damage and Complications}

During mapping of the epicardium (Figure 5), the same techniques employed during endocardial mapping are performed (e.g., pacemapping, entrainment, and electroanatomic mapping). However, prior to delivering radiofrequency energy on the epicardial surface, the regional anatomy must be carefully considered for potential collateral damage. Irrigated ablation technology has been shown to improve lesion size in the epicardial space compared to standard catheters. ${ }^{23}$ Acute and chronic coronary arterial damage with radiofrequency delivered overlying and adjacent to the vasculature have been shown in an animal model. ${ }^{24}$ The consensus statement recommends a distance greater than $5 \mathrm{~mm}$ from a coronary artery for safe ablation throughout all phases of the cardiac cycle, although many centers implement a larger safety margin of $1 \mathrm{~cm} .{ }^{25}$ Real-time integration of computed tomography imaging-derived coronary anatomy with electroanatomic mapping has been shown to be feasible after endocardial mapping. ${ }^{26}$

When ablation is performed on the lateral wall of the epicardial left ventricle, the course of the left phrenic nerve should be assessed. High output pacing (> $10 \mathrm{~mA})$ is useful to characterize the course of the phrenic nerve on electroanatomic mapping. ${ }^{27}$ If a targeted region is in close proximity to the phrenic nerve, separation of the epicardium from the parietal pericardium can be achieved by several methods, including instillation of water and air and displacement with a balloon. ${ }^{28,29}$

Epicardial fat presents two unique challenges for mapping and ablation of ventricular tachycardia. As fat insulates myocardium during contact mapping, low-voltages regions are frequently detected and can be difficult to distinguish from scar. ${ }^{30}$ Additionally, when ablation is required in these regions, interposed fat impairs the efficacy of radiofrequency delivery. 

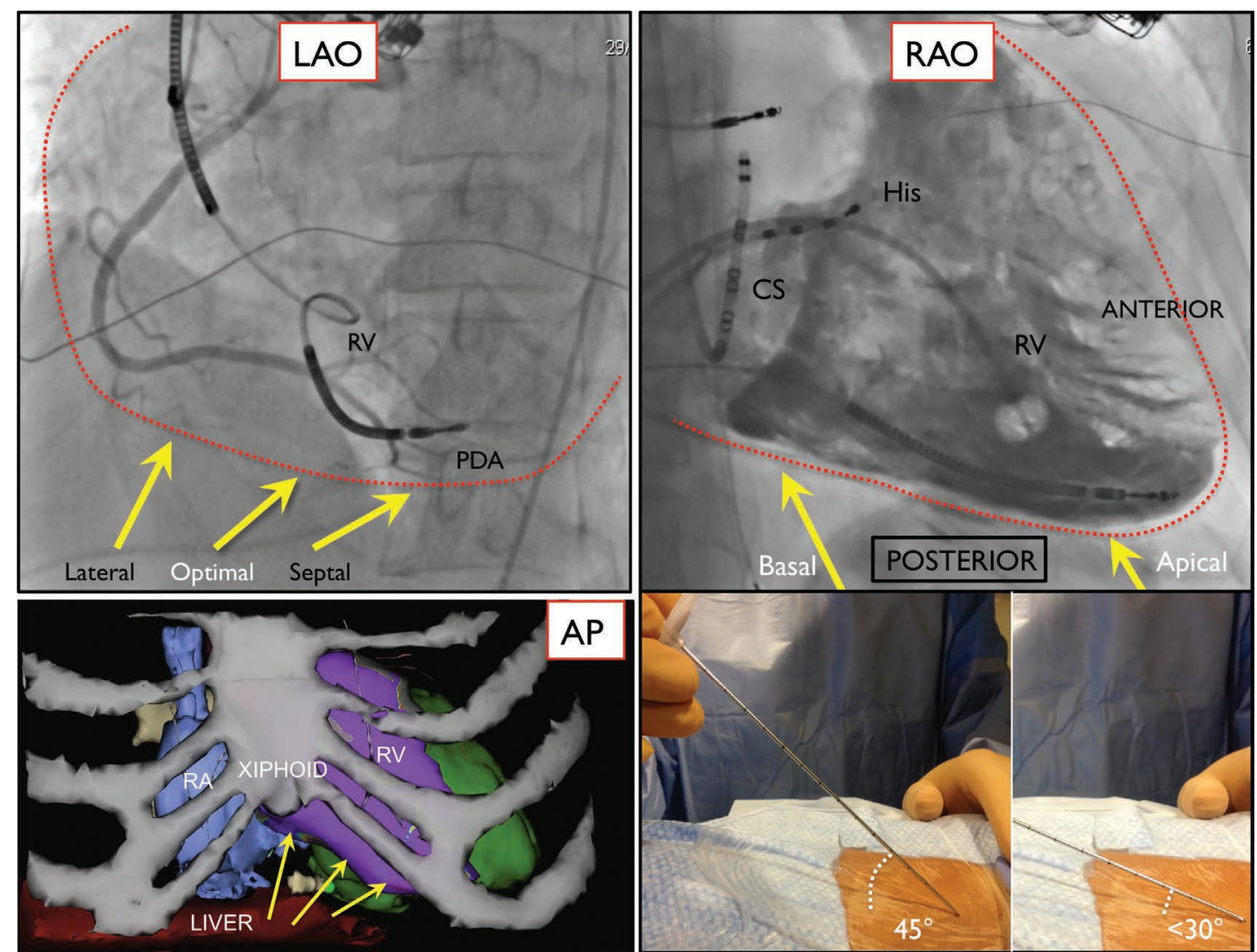

Figure 3. Fluoroscopic views of the angle of approach for subxiphoid epicardial access. In the LAO view, a tangential approach is preferable to minimize the risk of right ventricular puncture. In the RAO view, a steeper angulation results in a more posterior and basal approach. ${ }^{17} \mathrm{RAO}$ : right anterior oblique; LAO: left anterior oblique.
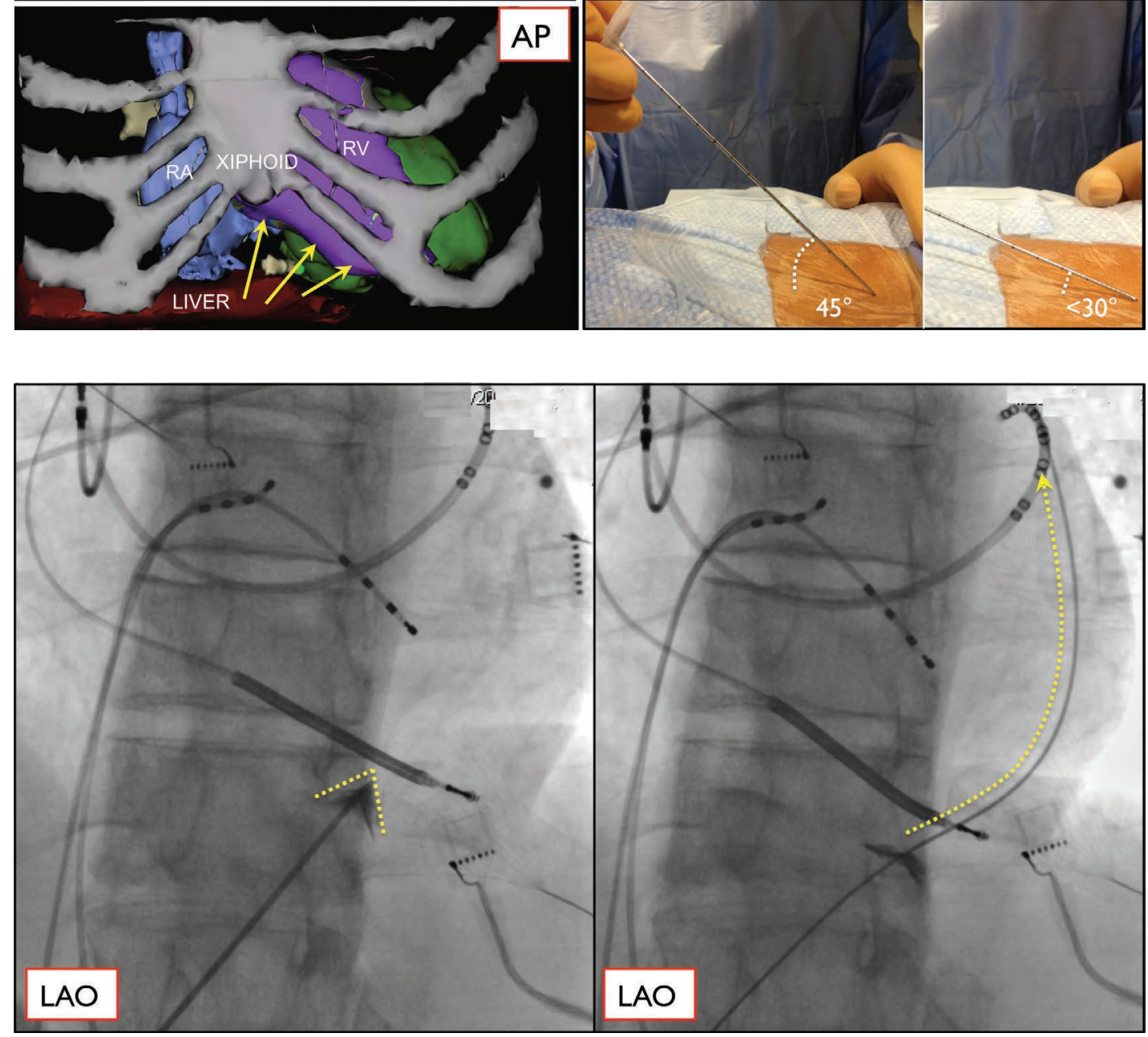

Figure 4. Fluoroscopic view of indentation of the pericardium prior to needle puncture with guidewire advanced toward the left heart border. ${ }^{17}$ LAO: left anterior oblique.

Symptomatic pericarditis is a common postprocedural complication that occurs in up to $30 \%$ of patients after epicardial mapping and ablation. In two large multicenter experiences, Sacher et al. described a 5\% (7 hemopericardium [> $80 \mathrm{cc}$ ] and 1 coronary stenosis) incidence of acute major complications out of 156 procedures, and Della Bella et al. observed major complications in 4\% (8 tamponade and 1 abdominal bleed) of 222 cases. $^{4,31}$ Among 109 epicardial procedures for ventricular tachycardia at our institution over a 7-year period, we observed a
$6 \%$ incidence of hemopericardium (> $80 \mathrm{cc}$ ), although no surgical intervention or procedural mortality was seen. ${ }^{14}$ Rare and unusual complications including right ventricular pseudoaneurysm, ventricular-abdominal fistula, liver puncture with intraabdominal bleed, hepatic subcapsular hematoma, and two cases of tamponade due to coronary sinus branch laceration have been also reported. ${ }^{32}$ Knowledge of pericardial anatomy, adjacent anatomic structures, and adverse effects is helpful in the avoidance and management of complications. 


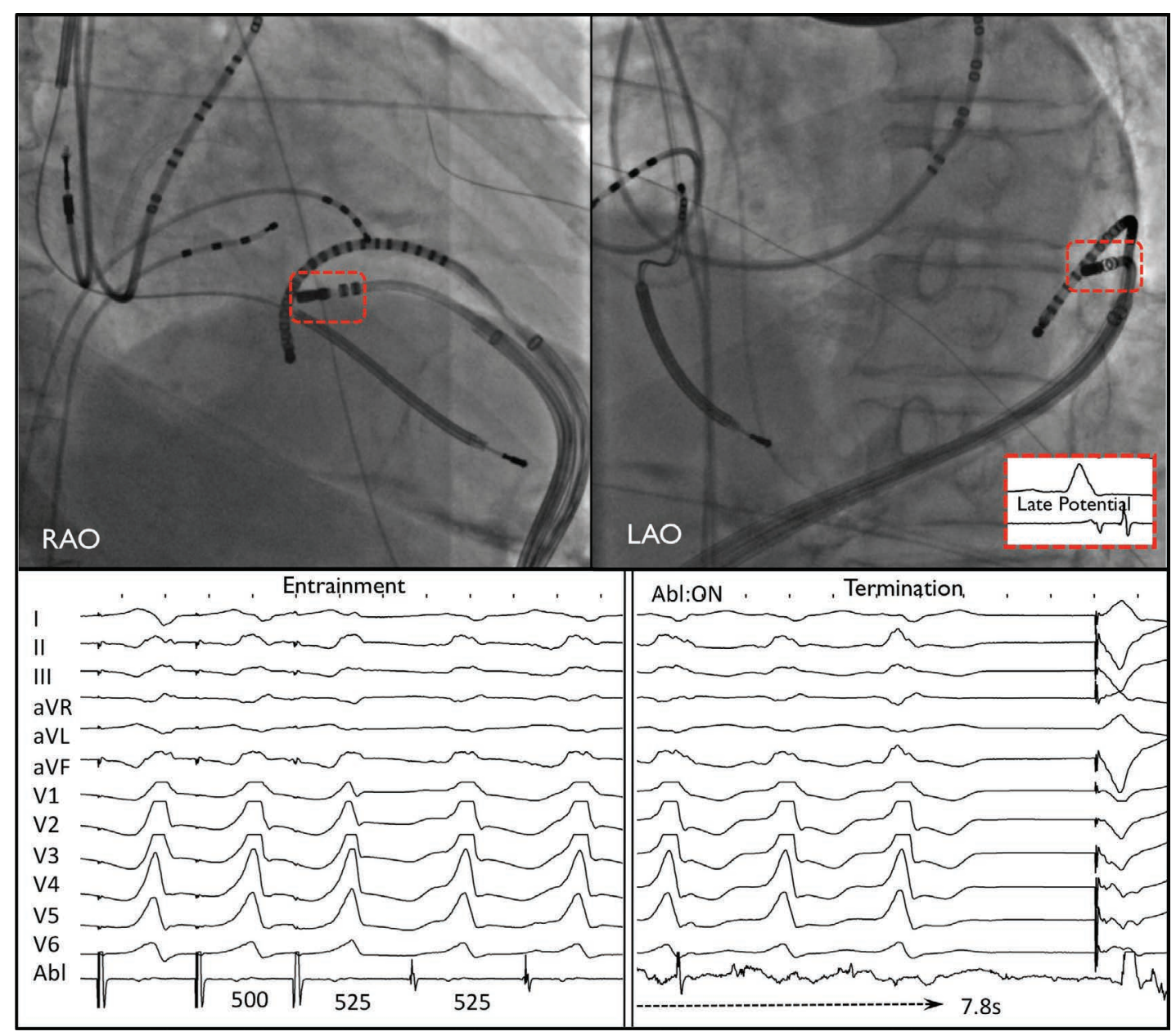

Figure 5. Fluoroscopic view of double access using a multipolar catheter and ablation catheter into the pericardial space in inferolateral scar. The late potential site during sinus rhythm becomes a mid-diastolic potential during ventricular tachycardia (VT), and entrainment mapping confirms an isthmus site. Ablation results in prompt termination of $\mathrm{VT} .{ }^{17} \mathrm{LAO}$ : left anterior oblique.

Conflict of Interest Disclosure: The authors have completed and submitted the Methodist DeBakey Cardiovascular Journal Conflict of Interest and Disclosure Statement and none were reported.

Keywords: ablation, ventricular, tachycardia, epicardial

\section{References}

1. Sosa E, Scanavacca M, d'Avila A, Pilleggi F. A new technique to perform epicardial mapping in the electrophysiology laboratory. J Cardiovasc Electrophysiol. 1996 Jun;7(6):531-6.

2. Sosa E, Scanavacca M, D’Avila A, Bellotti G, Pilleggi F. Radiofrequency catheter ablation of ventricular tachycardia guided by nonsurgical epicardial mapping in chronic Chagasic heart disease. Pacing Clin Electrophysiol. 1999 Jan;22(1 Pt 1):128-30.

3. Sosa E, Scanavacca M, d'Avila A, Oliveira F, Ramires JA. Nonsurgical transthoracic epicardial catheter ablation to treat recurrent ventricular tachycardia occurring late after myocardial infarction. J Am Coll Cardiol. 2000 May;35(6):1442-9.

4. Della Bella P, Brugada J, Zeppenfeld K, et al. Epicardial ablation for ventricular tachycardia: a European multicenter study. Circ Arrhythm Electrophysiol. 2011 Oct;4(5):653-9.

5. Boyle NG, Shivkumar, K. Epicardial interventions in electrophysiology. Circulation. 012 Oct 2;126(14):1752-69.

6. Reimer KA, Lowe JE, Rasmussen MM, Jennings RB. The wavefront phenomenon of ischemic cell death. 1. Myocardial infarct size vs duration of coronary occlusion in dogs. Circulation. 1977 Nov;56(5):786-94
7. Josephson ME, Simson MB, Harken AH, Horowitz LN, Falcone RA. The incidence and clinical significance of epicardial late potentials in patients with recurrent sustained ventricular tachycardia and coronary artery disease. Circulation. 1982 Dec;66(6):1199-204.

8. Bai R, Di Biase L, Shivkumar K, et al. Ablation of ventricular arrhythmias in arrhythmogenic right ventricular dysplasia/ cardiomyopathy: arrhythmia-free survival after endo-epicardial substrate based mapping and ablation. Circ Arrhythm Electrophysiol. 2011 Aug;4(4):478-85.

9. Cano O, Hutchinson M, Lin D, et al. Electroanatomic substrate and ablation outcome for suspected epicardial ventricular tachycardia in left ventricular nonischemic cardiomyopathy. J Am Coll Cardiol. 2009 Aug 25;54(9):799-808.

10. Dukkipati SR, d'Avila A, Soejima K, et al. Long-term outcomes of combined epicardial and endocardial ablation of monomorphic ventricular tachycardia related to hypertrophic cardiomyopathy. Circ Arrhythm Electrophysiol. 2011 Apr;4(2):185-94.

11. Santangeli P, Di Biase L, Lakkireddy D, et al. Radiofrequency catheter ablation of ventricular arrhythmias in patients with hypertrophic cardiomyopathy: safety and feasibility. Heart Rhythm. 2010 Aug;7(8):1036-42.

12. Soejima K, Stevenson WG, Sapp JL, Selwyn AP, Couper $G$, Epstein LM. Endocardial and epicardial radiofrequency ablation of ventricular tachycardia associated with dilated cardiomyopathy: the importance of low-voltage scars. J Am Coll Cardiol. 2004 May 19;43(10):1834-42. 
13. Di Biase L, Santangeli P, Burkhardt DJ, et al. Endo-epicardial homogenization of the scar versus limited substrate ablation for the treatment of electrical storms in patients with ischemic cardiomyopathy. J Am Coll Cardiol. 2012 Jul 10;60(2):13241.

14. Tung R, Michowitz Y, Yu R, et al. Epicardial ablation of ventricular tachycardia: an institutional experience of safety and efficacy. Heart Rhythm. 2013 Apr;10(4):490-8.

15. Yoshiga Y, Mathew S, Wissner E, et al. Correlation between substrate location and ablation strategy in patients with ventricular tachycardia late after myocardial infarction. Heart Rhythm. 2012 Aug;9(8):1192-9.

16. Schmidt B, Chun KR, Baensch D, et al. Catheter ablation for ventricular tachycardia after failed endocardial ablation: epicardial substrate or inappropriate endocardial ablation? Heart Rhythm. 2010 Dec;7(12):1746-52.

17. Huang SKS, Miller J, editors. Catheter ablation of cardiac arrhythmias. 3rd ed. Philadelphia: Elsevier Health Sciences; 2014. 800 p.

18. Baudoin YP, Hoch M, Protin XM, Otton BJ, Ginon B, Voiglio EJ. The superior epigastric artery does not pass through Larrey's space (trigonum sternocostale). Surg Radiol Anat. 2003 JulAug;25(3-4):259-62.

19. Mizuno H, Maccabelli G, Della Bella P. The utility of manually controlled steerable sheath in epicardial mapping and ablation procedure in patients with ventricular tachycardia. Europace. 2012 Aug;14 Suppl 2:ii19-ii23.

20. Tschabrunn CM, Haqqani HM, Cooper JM, et al. Percutaneous epicardial ventricular tachycardia ablation after noncoronary cardiac surgery or pericarditis. Heart Rhythm. 2013 Feb;10(2):165-9.

21. Soejima K, Couper G, Cooper JM, Sapp JL, Epstein LM, Stevenson WG. Subxiphoid surgical approach for epicardial catheter-based mapping and ablation in patients with prior cardiac surgery or difficult pericardial access. Circulation. 2004 Sep 7;110(10):1197-201.

22. Michowitz Y, Mathuria N, Tung R, et al. Hybrid procedures for epicardial catheter ablation of ventricular tachycardia: value of surgical access. Heart Rhythm. 2010 Nov;7(11):1635-43.

23. D'Avila A, Houghtaling C, Gutierrez P, et al. Catheter ablation of ventricular epicardial tissue: a comparison of standard and cooled-tip radiofrequency energy. Circulation. 2004 May 18;109(19):2363-9.
24. D'Avila A, Gutierrez P, Scanavacca M, et al. Effects of radiofrequency pulses delivered in the vicinity of the coronary arteries: implications for nonsurgical transthoracic epicardial catheter ablation to treat ventricular tachycardia. Pacing Clin Electrophysiol. 2002 Oct;25(10):1488-95.

25. Aliot EM, Stevenson WG, Almendral-Garrote JM, et al.; European Heart Rhythm Association (EHRA); Registered Branch of the European Society of Cardiology (ESC); Heart Rhythm Society (HRS); American College of Cardiology (ACC); American Heart Association (AHA). EHRA/HRS Expert Consensus on Catheter Ablation of Ventricular Arrhythmias: developed in a partnership with the European Heart Rhythm Association (EHRA), a Registered Branch of the European Society of Cardiology (ESC), and the Heart Rhythm Society (HRS); in collaboration with the American College of Cardiology (ACC) and the American Heart Association (AHA). Heart Rhythm. 2009 Jun;6(6):886-933.

26. van Huls van Taxis CF, Wijnmaalen AP, Piers SR, van der Geest RJ, Schalij MJ, Zeppenfeld K. Real-time integration of MDCT-derived coronary anatomy and epicardial fat: impact on epicardial electroanatomic mapping and ablation for ventricular arrhythmias. JACC Cardiovasc Imaging. 2013 Jan;6(1):42-52.

27. Fan R, Cano O, Ho SY, et al. Characterization of the phrenic nerve course within the epicardial substrate of patients with nonischemic cardiomyopathy and ventricular tachycardia. Heart Rhythm. 2009 Jan;6(1):59-64.

28. Buch E, Vaseghi M, Cesario DA, Shivkumar K. A novel method for preventing phrenic nerve injury during catheter ablation. Heart Rhythm. 2007 Jan;4(1):95-8.

29. Di Biase L, Burkhardt JD, Pelargonio G, et al. Prevention of phrenic nerve injury during epicardial ablation: comparison of methods for separating the phrenic nerve from the epicardial surface. Heart Rhythm. 2009 Jul;6(7):957-61.

30. Tung R, Nakahara S, Ramirez R, Lai C, Fishbein MC, Shivkumar $K$. Distinguishing epicardial fat from scar: analysis of electrograms using high-density electroanatomic mapping in a novel porcine infarct model. Heart Rhythm. 2010 Mar;7(3):389-95.

31. Sacher F, Roberts-Thomson K, Maury P, et al. Epicardial ventricular tachycardia ablation a multicenter safety study. J Am Coll Cardiol. 2010 May;55(21):2366-72.

32. Koruth JS, Aryana A, Dukkipati SR, et al. Unusual complications of percutaneous epicardial access and epicardial mapping and ablation of cardiac arrhythmias. Circ Arrhythm Electrophysiol. 2011 Dec;4(6):882-8. 